RESEARCH REPORT

\title{
COMPARATIVE STUDY ON THE EFFICACY OF FRENKEL'S AND BALANCING EXERCISES IN PATIENTS WITH CEREBELLAR DYSFUNCTION
}

\begin{abstract}
OBJECTIVE

To compare the effectiveness of Frenkel's exercise and balancing exercises in patients with cerebellar dysfunction

STUDY DESIGN

Experimental Study

STUDY SETTING \& PARTICIPANTS

The study has been conducted in a tertiary care hospital. Sample size of 20 patients has been drawn from the population having ataxia, and they were equally divided into two groups. Subjects were randomly assigned to each group.

INTERVENTION

One group was given Frenkel's exercise regime and another has been treated by balancing exercises.

\section{OUTCOME MEASURES}

Assessment was performed to find out the pre and post score on the basis of the Scale for the Assessment and Rating of Ataxia (SARA).

\section{RESULTS}

There was a significant difference in sensation, anterior balance, gait framework, and expanded degree of disability in a post treatment data. A meaningful difference was observed in patients who received frankel's exercise regime. There was also a statistically compelling difference in pendular movements and dysdiadochokinesia.

\section{CONCLUSION}

According to our study, physiotherapy approaches were purpose fully effective to decrease the ataxia. In cerebellar dysfunction rehabilitation, combination of relevant physical therapy technique is also found potent in recovery.
\end{abstract}

\section{KEYWORDS}

Frankel's Exercises, Balancing Exercise, Ataxia, Cerebellar Dysfunction, Dysdiadochokinesia, Pendular Movements

\author{
Qurat ul Ain Adnan \\ Senior Lecturer \\ Ziauddin College of Physical Therapy \\ Ziauddin University \\ aqua_8428@hotmail.com \\ Hassan Abbas Rizvi \\ Associate Professor, Principal \\ Liaquat National School of \\ Physical Therapy \\ Liaquat National Hospital \\ hassan.abbas@Inh.edu.pk

\section{Deepak Kumar} \\ DPT Student \\ Ziauddin College of Physical Therapy \\ Ziauddin University

\section{Aitezaz Idrees Raja} \\ DPT Student \\ Ziauddin College of Physical Therapy \\ Ziauddin University

\section{Rida Naqvi} \\ DPT Student \\ Ziauddin College of Physical Therapy \\ Ziauddin University

\section{Yumna Ali} \\ DPT Student \\ Ziauddin College of Physical Therapy \\ Ziauddin University
}

[Adnan QA, Rizvi HA, Kumar D, Raja Al, Naqvi R, Ali Y. Comparative Study on the Efficacy of Frenkel's and Balancing Exercise in Patients with Cerebellar Dysfunction. Pak. j. rehabil. 2016;5(2):37-42] 


\section{INTRODUCTION}

The development of human being starts with motor movements that cause neural development that follows birth. Once the development of motor system is done, the ability of maintaining balance in different positions is gained that leads to neuromuscular coordination'.

Normal muscle tone allows the individual to stand straight against gravity. It helps to maintain the change and provide resistance to stretch. Balance is stated as having weight equally or the ability to maintain a position without causing fall. Contrary the muscular coordination is controlling the multi joint movement that needs coordination in appropriate manner. Individual required muscle tone, balance and muscular coordination to perform activities of daily living.

Postural control is usually affected by Nervous system injuries. Conditions like Stroke, Head injuries, Ataxias, Multiple Sclerosis (MS), Parkinson and Sensory neuropathies periodically suffer from balance and coordination issues. Some diseases have balance as a prevalent problem, whereas coordination problems are focused in others. When intimately studied, many patients observed with existence of both problem².

Incoordination of movements and improper postural control is a movement disorder with balance and walking disturbances which is known as ataxia. Ataxia is a Greek word which means 'without order'. It is most typically defined as the incoordination of movements ${ }^{3}$. However, this definition is incompetent in proving ataxia. Ataxia present as faulty postural control and crudeness of multi-joint movements ${ }^{4,5}$. Ataxia cannot be considered in isolation from balance dysfunctions although it is followed by incoordination and gait problems; in some cases, balance dysfunctions observed as muscle weaknesses in the absence of faulty movements ${ }^{6}$. For instance, perfectly timed sequence of muscle activity can be considered as normal walking that is a feedback to both internal and external forces, and then it is easy to consider walking as an effort depending on coordination as well as on balance ${ }^{7}$.

Thus, in-coordination and balance dysfunction in movements without muscle weakness is a more precise definition of ataxia $^{8}$.

Postural disequilibrium results from the deficiency in postural control and leads to balance dysfunctions. Sensory, cerebellar and vestibular ataxia and frontal ataxia are the subtypes of this disease. Mixed ataxia is defined as combination of at-least two basic types of ataxia. Various diseases, diverse clinical manifestation, interference of neurological structures appear as ataxia. Physical treatment applications are of great importance as ataxia is defiant to medical cure. Treatment protocols for the improvement of functional task impaired in Ataxia includes, Proprioceptive training, balance exercises, stabilization techniques and vestibular exercises?. Supportive devices are also used as redeeming operation. Restoration of the ataxic patients with sensory ataxia, peripheral neuropathy is common. In sensory ataxia patient can balance and coordinate movements with open eyes but when they are implied the patient cannot balance himself because no information is received from the peripheral nervous system ${ }^{10}$.

Splitting sensory input and transforming the information with failure of balance is associated with sensory ataxia. It is a slowly progressive condition if not treated; it can be acquired due to some disease or can be ingrained. Root cause should be treated and managed for the recovery of ataxia ${ }^{10,11}$.

Four unrelated patients were studied with a relentless sensory ataxia in association with dysarthria and chronic progressive external Ophthalmoplegia. Electro-physiologic and pathologic studies showed serious axonal loss affecting sensory nerves. Various mitochondrial DNA deletions in muscle and nerve were found in Molecular genetic analysis. Sensory ataxic neuropathy may be the prevailing and presenting indication of a mitochondrial derangement, differential diagnosis should encompass mitochondrial etiology. The triad of sensory ataxic neuropathy, dysarthria, and Ophthalmoparesis (SANDO) may represent a unique mitochondrial disease related with numerous mitochondrial DNA deletions ${ }^{12}$.

Another study which says, Friedreich ataxia (FRDA), the most widely recognized subsiding ataxia, is plot by debased of the tactile neurons of the spinal Cord. It is brought about by lessened levels of frataxin; a protein required in the biosynthesis of iron-sulfur bunch, two mouse models for FRDA were created through a spatiotemporally controlled contingent quality focusing on methodology. They particularly create dynamic blended cerebellar and tactile ataxia. Histological studies demonstrated spinal rope and dorsal root ganglia peculiarities with non-appearance of motor neuropathy, an exemplary sign of the human illness. Furthermore, a cerebellar granule cell loss, Purkinje cell arborization defects were exposed. The resourceful obsessive component in the DRG was a self-consumption mechanism, prompting removal of mitochondrial rubble and development of lipofuscin stores. These mice represent brilliant models for FRDA to resolve the neurotic deluge and to test structure that hamper with the decaying procedure ${ }^{13}$.

Comparative examination was done to ask whether ataxia in Miller Fisher disorder (MFS) is brought by the loss of proprioception or cerebellar brokenness, 
we concentrated on the force range pinnacle of the body influence recurrence in 10 MFS patients, and contrasted the outcomes and those of patients with cerebellar or tangible ataxia. The cerebellar patients had a crest at $2.4 \mathrm{~Hz}$, while tactile ataxia patients had a $1-\mathrm{Hz}$ crest. Nine of the MFS patients had an unmistakable $1-\mathrm{Hz}$ crest. Clinical tangible misfortune or anomalous tactile nerve possibilities were available in just 3 patients, while soleus $\mathrm{H}$-reflexes were missing in every one of the MFS patients. MFS patients have brokenness of the proprioceptive afferent framework, and the unique tactile ataxia might be created by the particular contribution of muscle axle afferents ${ }^{14}$.

In the young adults Multiple sclerosis (MS) is a prevailing progressive neurological disorder ${ }^{15}$. Cerebellum is one of the area affected in MS. Derangement in this structure leads to ataxia and shortcoming. Exercise is a competent method to reduce functional disorders in MS patients ${ }^{16}$. This study presents the potency of Frankel's exercises in improvement of status in cerebellar dysfunction patients.

\section{METHODOLOGY}

\section{Population and Study Sample}

Sample was drawn out randomly from the population of patients who were having cerebellar ataxia.

\section{Sample Size}

20 patients

\section{Study Design}

Experimental study

\section{Study Period}

This study was conducted for five months

\section{Sources of Data}

A tertiary care hospital

\section{Collection of Data}

During complete tenure of our data collection we have experienced so many different scenarios and got the chance to meet different people, it was an encouraging and very learning experience for us to collect the data. Some circumstances were motivating and mobilizing, some situations were difficult to handle. Unfortunately some patients need to back out during research due to any problem, or relapse of the disease. It took almost 4 months to collect data.

\section{Data Analysis Strategies}

To analyze the data and find the differences in both groups, paired-T test was applied on the gathered data through SPSS version 20. It was helpful in finding diversity in both groups.

\section{Measurement and Assessment}

The main aim of physiotherapy and the rehabilitation team for the patients of ataxia is to enhance the quality of treatment and it can be achieved by using appropriate measures, assessment tools and strategies. Treatment is not only the mainstay for Ataxic patients but follow up treatment should include getting the better results. Observation over the period of time significantly effects the treatment. Different approaches are applied to assess ataxia. Any problem in measurement and assessment turned out to be more upsetting in instances of ataxia ${ }^{17}$. There are different approaches for improving proprioception, balance and extremity ataxia. Supportive devices, sport activities and vestibular exercise reduce the severity of ataxia.

\section{Inclusion Criteria}

- Male patients who have Balancing problems

- Age between 40-60 years

\section{Exclusion Criteria}

- Females patients

- Neurological symptoms, other than ataxia

\section{Procedure \& Intervention}

20 people who voluntarily involved in this study were drawn from out-patient department of physical therapy of a tertiary care hospital. Demographic data e.g., gender, age were recorded. We provided participants information sheet to the entire patients to give them knowledge about our study and to encourage their voluntary participation before commencement of study.

All patients were given an informed consent before participation. We performed a basic assessment to find out the pre score on the basis of Scale for the assessment and rating of ataxia (SARA) ${ }^{18}$. Participants were divided into two groups. First group was treated with balancing exercises and the second group was treated with the Frankel's exercises following sitting, lying and walking protocol.

\section{RESULTS}

All of the subjects considered to complete their prescribed exercise regime, some patients withdrawn from research process. A contemporary way to deal with stride issue and restoration is the utilization of systemic investigations and assignment of particular locomotors worldview.

By specific and focused treatment we would be able to improve daily activities and life pattern of patients.

$p$ value of less than 0.05 , is considered to be significant result using paired-T-test.

The pre-exercise mean value of balancing exercise 
was $35.30 \pm 3.62$ and post exercise mean value was $32.40 \pm 3.51$ with the $p$ value of $<0.01$, while the pre-exercise mean value of Frenkel's exercise was $33.20 \pm 3.69$ and post exercise mean value was $17.05 \pm 4.71$ with the $p$ value of $<0.01$.

\begin{tabular}{|c|c|c|c|}
\hline \multicolumn{4}{|c|}{ Frankel's and Balancing Exercise Regime } \\
\hline & $\begin{array}{l}\text { PRE- } \\
\text { EXERCISE } \\
\text { MEAN } \pm S D\end{array}$ & $\begin{array}{l}\text { POST- } \\
\text { EXERCISE } \\
\text { MEAN } \pm S D\end{array}$ & p-value \\
\hline $\begin{array}{l}\text { BALANCING } \\
\text { EXERCISE }\end{array}$ & $35.30 \pm 3.62$ & $32.40 \pm 3.51$ & $<0.01$ \\
\hline $\begin{array}{l}\text { FRANKEL'S } \\
\text { EXERCISE }\end{array}$ & $33.20 \pm 3.69$ & $17.05 \pm 4.71$ & $<0.01$ \\
\hline
\end{tabular}

\section{Difference between Pre-Exercise and Post Exercise Score of patients received Frenkel's Regime}

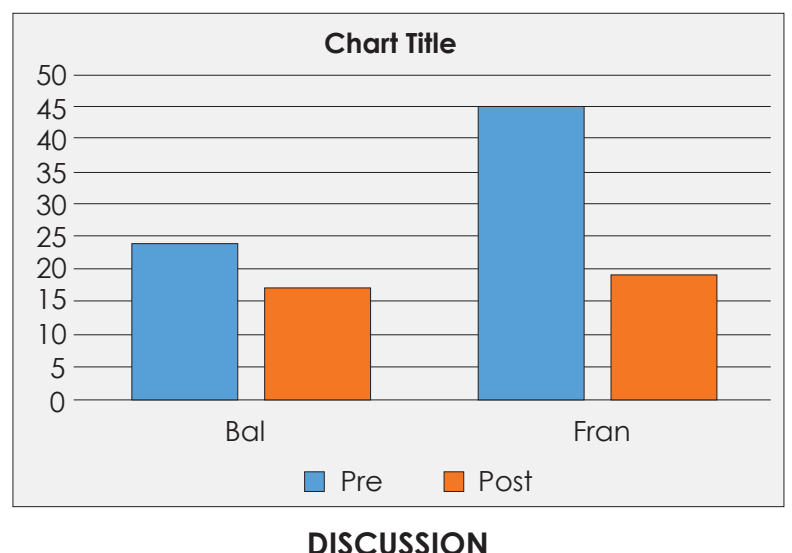

Ataxia is the disorder that affects the balance and stability. Several studies are done to evaluate the conditions that disturb balance and stability ${ }^{19}$. Treatment regime includes exercises that are designed for the management of extremity that is affected and is in ataxia, are used to offer fixation by forming balance between the eccentric and concentric state of muscle contractions within the multi-joint activities of lower limbs and the upper limbs in particular. During the enactment of the exercises, it is significant to establish gentle, controlled and reciprocal joint movements and balance. Frankel's coordination exercises are developed for the purpose of balance and stability in ataxic patients ${ }^{3}$. A randomized control trail was done to investigate the effect of an additional vestibular stimulated exercise programme on balance for patients with benign paroxysmal positional vertigo ${ }^{20,21}$. For this purpose, static balance tests, tandem walk test, Dynamic Gait Index and subjective rating of the intensity of vertigo were measured at baseline, two-week and four-week assessments. It demon- strated that additional exercise training, which emphasizes vestibular stimulation, can improve balance ability and functional gait performance among patients with benign paroxysmal positional vertigo who had already undergone the canalith repositioning manoeuvre ${ }^{6}$.

But the drawback of this study is that it only worked on improvement of balance in patients with benign paroxysmal positional vertigo involving posterior canal. Some researchers assessed Static balance through External Perturbation Tests by Push and Release test ${ }^{22}$ or Pull test and Static and dynamic balance through Sensory Integration Test of Computerized Dynamic Posturography ${ }^{23,24}$. Another study was done to improve gait and balance impairment in early multiple sclerosis in the absence of clinical disability. Ten MS patients with mild pyramidal signs, $10 \mathrm{MS}$ patients with no pyramidal signs and 20 age- and gender-matched control subjects were assessed using laboratory-based gait analysis and clinical balance measures. Both MS groups demonstrated reduced speed and stride length, and prolonged double limb support, compared to the control group, along with alterations in the timing of ankle muscle activity, and the pattern of ankle motion during walking, which occurred independent of gait speed. The identification of incipient gait and balance impairment in MS patients with recent disease onset suggests that motor function may begin to deteriorate in the early stages of the disease, even in the absence of clinical signs of pyramidal dysfunction ${ }^{25}$.

By evaluating the results of the studies the need of our study emerged to differentiate between the ataxia patients receiving Frankel's regime and patients receiving other regime with no particular considerations of level of disability. Sample receiving Frankel's exercises have showed consistent improvement. Researchers worked on different scales that are simple and validated method for assessing cerebellar ataxia over a wide range of severity ${ }^{24}$ and the assessment of this study has been made through a common scale (SARA) ${ }^{18}$ which is a great tool to evaluate the treatment regime according to the needs of patients.

The result was somehow expected because improvement has been seen from the treatment. Through this study we found out that those patients who received proper treatment regime showed better results in their level of disabilities and early results than those who have been taken normal non specified treatment regime. The level of disability do differ for all the patients.

The result was collected and compiled very carefully, so that chances of mistake could be lessen as much as possible. As a suggestion we would recommend other researchers to use different scales, or take only one type of ataxia so that it could help us 
all to asses and treat patients considering specific type of ataxia.

The major drawback of this research was the lack of resources. Many of the patients were assessed from private hospitals with proper physiotherapy and rehabilitation setups with international standards. Our colleagues and co-workers supported us during this process, specially the families who showed their thorough interest in our project, helped us by all means.

This study should be replicated to ensure the effects of treatment that would work in favor of patients, their families and health professionals. Although it was a time taking process but majority of the patients showed great improvement with continuous physiotherapy. Assessment of the patient, proper diagnosis, determination of appropriate treatment procedures and problem solving methodology with proper implementation of treatment goals regularly is vital for the success of treatment regime. Frankel's exercises seemed effected in our study as compared to general exercises in patients with ataxia. Furthermore ataxia can be studied with respect to problems persist in cerebellum and other brain areas to know specific region so that specific treatment could planned. It is therefore suggested that patients with ataxia should be studied further for better understanding and awareness of the condition.

\section{CONCLUSION}

Cerebellar ataxia is a neurological problem that affects majorly on movements and is sometimes more obstinate and challenging to cope up with. The point of this study was to differentiate between the adequacies of treatment. To follow a particular regime focusing on the particular effected area, treatment should be as focused as addressing only to the problem creating areas.

This study was to differentiate between the specific tasks oriented regime and an overall rehabilitation program. After this study we came to know how much frenkel's exercise regime was effective in treating patients with cerebellar ataxic gait abnormality.

\section{REFERENCES}

[1] Buatois S, Miljkovic D, Manckoundia P, et al. 2008. Five times sit to stand test is a predictor of recurrent falls in healthy community-living subjects aged 65 and older. J Am Geriatr Soc. 2008:56(8):1575-7

[2] Buatois S, Gueguen R, Gauchard GC, et al. Post urography and risk of recurrent falls in healthy non-institutionalized persons aged over 65 . Gerontology 2006;52(6):345-352
[3] Armutlu K. Ataxia: Physical Therapy and Rehabilitation Applications for Ataxic Patients. International Encyclopedia of Rehabilitation. 2010. Available from: http://cirrie.buffalo.edu/encyclopedia/en/article/112/

[4] Topka H, Konczak J, Dichgans J. Coordination of multi-joint arm movements in cerebellar ataxia: analysis of hand and angular kinematics. Exp Brain Res. 1998; 1 19(4):483-492

[5] Cham R, Perera S, Studenski SA, Bohnen NI. Striatal dopamine denervation and sensory integration for balance in middle-aged and older adults. Gait and Posture. 2007;26(4):516-525

[6] Chang WC, Yang YR, Hsu LC, Chern CM, Wang RY. Balance improvement in patients with benign paroxysmal positional vertigo. Clin Rehabil. 2008;22(4):338-47

[7] Chaudhry H, Findley T, Quigley KS, Ji Z, Maney M, Sims $T$ et al. Measures of postural stability. Journal of Rehabilitation Research and Development. 2004;41 (5):713-720

[8] Borello-France DF, Whitney SL, Herdman SJ et al. Assessment of vestibular hypofunction, in Vestibular Rehabilitation 1994;247-286

[9] Adler SS, Beckers D, Buck M. PNF in practice. Heidelberg: Springer. 2000

[10] Armutlu K, Karabudak R, Nurlu G. Physiotherapy approaches in the treatment of ataxic multiple sclerosis: a pilot study. Neuro rehabil Neural Repair. 2001;15(3):203-211

[11] Marmolino D, Manto M. Past, Present and Future Therapeutics for Cerebellar Ataxias. Curr Neuropharmacol. 2010; 8(1): 41-61

[12] Assadi M, Leone P, Veloski JJ Schwartzman RJ, Janson CG, Campellone JV. Validating an Ataxia Functional Composite Scale in spin cerebellar ataxia. J Neurol Sci. 2008;268(1-2):136-9

[13] Brodal, A. Neurological Anatomy in Relation to Clinical Medicine, ed. 2, New York, Oxford University Press, 1969

[14] Bertoti DB. Effect of therapeutic horseback riding on posture in children with cerebral palsy. Phys Ther. 1988;68(10):1505-1512

[15] Blennerhassett JM, Jayalath VM. The Four Square Step Test is a feasible and valid clinical test of dynamic standing balance for use in ambulant people post stroke. Arch Phys Med Rehabil. 2008:89(11):2156-2161

[16] Cattaneo D, Jonsdottir J, Zocchi M, Regola A. Effects of balance exercises on people with multiple sclerosis: a pilot study. Clin Rehabil. 2007;21 (9):771-778

[17] Danek A. [On the vestiges of Heinrich Frenkel (1860-1931)--Pioneer of Neurorehabilitation. Annotation to the cover picture]. Nervenarzt. 2004; 75(4):41 1-413

[18] Schmitz-Hubsc T, du Montcel ST, Baliko L, Berciano J, Boesch S, Depondt C et al. Scale for the assessment and rating of ataxia: development of a new clinical scale. Neurology. 2006;66(11):1717-1720 
[19] Diracoglu D, Aydin R, Baskent A, Celik A. Effects of kinesthesia and balance exercises in knee osteoarthritis. J Clin Rheumatol. $2005 ; 11(6): 303-10$

[20] Dix MR. The rationale and technique of head exercises in the treatment of vertigo. Acta Otorhinolaryngolica Belgica. 1979;33(3):370-384

[21] Corna S, Nardone A, Prestinari A, Galante M, Grasso M, Schieppati M. Comparison of Cawthorne-Cooksey exercises and sinusoidal support surface translations to improve balance in patients with unilateral vestibular deficit. Arch Phys Med Rehabil. 2003;84(8):1173-1184

[22] Du Montcel ST, Charles P, Ribai P, Goizet C, Le Bayon A, Labauge P et al. Composite cerebellar functional severity score: validation of a quantitative score of cerebellar impairment. Brain. 2008;131 (5):1352-61

[23] Crutchfield CA, Shumway-Cook A, Horak FB. Balance and coordination training. In: Scully RM, Barnes MR, editors. Physical Therapy. Philadelphia: Lippincott Company. 1989

[24] D'Abreu A, Franca MJ, Lopes-Cendes I, et al. The international cooperative ataxia rating scale in Machado-Joseph disease. Comparison with the unified multiple systems atrophy rating scale. Movement Disorders. 2007;22(15): 2162-2169

[25] Doring A, Pfuveller CF, Paul F, Dorr J. Exercise in multiple sclerosis-an integral component of disease management. EPMA J. 2011;3(1):2 